\title{
Interactions between Antitumor Alkylphosphocholines and Membrane Sphingolipids in Langmuir Monolayers
}

\author{
A. WNĘTRZAK ${ }^{a}$, K. EĄTKA ${ }^{a}$ AND P. DYNAROWICZ-EĄTKA ${ }^{b, *}$ \\ ${ }^{a}$ Institute of Physics, Jagiellonian University, W.S. Reymonta 4, 30-059 Kraków, Poland \\ ${ }^{b}$ Faculty of Chemistry, Jagiellonian University, R. Ingardena 3, 30-060 Kraków, Poland
}

\begin{abstract}
Alkylphosphocholines (APCs) are new generation, highly selective antineoplastic drugs, whose mechanism of action is not fully understood. It is known that in contrast to traditional chemotherapeutics, APCs do not induce cell death by apoptosis or necrosis as a result of DNA damage, but target cellular membranes and affect their biophysical properties. However, it is still unknown which membrane component attracts APC molecules selectively to cancer cells. In order to get insight into this issue, systematic investigations on the interactions between APCs and particular membrane components are highly required. Such experiments can be performed with the Langmuir monolayer technique, serving as a biomembrane model. Because of overexpression of gangliosides in tumor progression and the ability of APCs to insert into membrane rafts, two sphingolipids, i.e. sphingomyelin $(\mathrm{SM})$ and ganglioside $\mathrm{GM}_{1}$ have been examined as potential membrane targets. In this respect, their interactions with three alkylphosphocholines, differing in their hydrophobic part: hexadecylphosphocholine (HePC), octadecylphosphocholine (OcPC) and erucylphosphocholine (ErPC) have been studied and the following systems have been analysed: $\mathrm{SM}$ (or GM1)/HePC, SM(or GM1)/OcPC and SM(or GM1)/ErPC. It was found that all the investigated APCs show strong affinity to ganglioside in contrast to sphingomyelin. Differences in interaction of APCs with both investigated sphingolipids were studied based on experimental surface pressure $(\pi)$ versus mean molecular area $(A)$ isotherms, and analyzed qualitatively (with mean molecular area values) as well as quantitatively (with $\Delta G^{\text {exc }}$ function). The obtained results have also been analysed taking into consideration geometry of interacting molecules. Our results suggest that gangliosides may be molecular targets for APCs, attracting them to tumor cells. Although the interactions with sphingomyelin were found to be unfavourable, further studies on more complex system, containing APCs mixed with sphingomyelin and cholesterol, are required to better understand the role of lipid rafts in the selectivity of APCs.
\end{abstract}

DOI: 10.12693 /APhysPolA.125.886

PACS: $68.18 .-\mathrm{g}$

\section{Introduction}

Synthetic antitumor lipids (ATLs) are new generation anticancer drugs, which were synthesized as metabolically stable analogues of natural lysolecithins [1]. Contrary to radiotherapy and many cytostatics used in chemotherapy, ATLs do not interfere with cellular DNA, but - due to their phospholipids-like structure - incorporate directly into biological membranes [2]. The group of synthetic antitumor lipids consists of two major types. One of them are alkyllysophospholipid analogues (ALPs), in the structure of which ester bonds in the $\mathrm{C} 1$ and $\mathrm{C} 2$ positions of the glycerol backbone are replaced by ether linkages, and the hydroxyl group in C2 position is transformed into a short-chained methoxy or ethoxy group. Edelfosine (1-O-octadecyl-2-O-methyl-rac-glycero-3-phosphocholine, in short Et-18- $\left.\mathrm{OCH}_{3}\right)$ is one of the most efficient drugs of this type, beside its thioether derivative - ilmofosine. Another group, known as alkylphosphocholines (APCs), are characterized by simplified structure, in which the glycerol backbone is lacking and substituted for a simple alkyl

*corresponding author; e-mail: ucdynaro@cyf-kr.edu.pl chain [3], as exemplified by hexadecylphosphocholine (miltefosine) [4], octadecylphosphocholine, perifosine and erucylphosphocholine [5].

Synthetic antitumor lipids, due to their phospholipid-like structure, interact with biomembranes [1, 2] and they have been found to be selective $[1,6]$. High selectivity of synthetic antitumor lipids is responsible for a great interest in phospholipid drugs in recent years as they could prevent oncological patients from many systemic side-effects, being a result of healthy tissues degradation by nonspecific cytotoxic therapies. It is well known that ATLs uptake and the effective dose are important in their biological and cytotoxic action, but it is still not clear how these ether lipids incorporate into the cell [1]. Three hypotheses have been put forward as regards molecular targets for ATLs.

Firstly, it has been postulated that the membrane fluidity plays an important role in selective incorporation of the drugs to cancer cells. Studies on leukemia cells showed that the cancerous membrane is more fluid as compared to that of normal cells, which results mainly from decreased total cholesterol and cholesterol-to-phospholipids molar ratio [7] and increased percentage of unsaturated fatty acids acyl chains of the major membrane phospholipids [8]. The same tendency was also observed for other cancerous cell lines (e.g. lung cancer) $[9]$, but for prostate [10] or breast cancer [11] the 
effect was opposite, i.e. malicious membrane was more rigid as it contained increased level of cholesterol. Although monolayer experiments proved strong affinity of APCs to cholesterol [12], recent studies [13] revealed that cholesterol and cholesterol-to-phospholipid ratio are not major determinants of ATLs sensitivity.

Second hypothesis holds that ATLs target two different subcellular structures in a cell type-dependent manner, namely cell surface lipid rafts in leukemic and lymphoma cells [14] and endoplasmic reticulum in solid tumor cells $[15,16]$.

Thirdly, gangliosides, which are overexpressed in tumor progression, have been supposed to target these drugs selectively to cancer cells [17].

The aim of present investigations was to understand more deeply the interactions between selected APCs (namely hexadecylphosphocholine, HePC; octadecylphosphocholine, OcPC; erucylphosphocholine, ErPC) (Fig. 1) and sphingolipids (sphingomyelin, SM, and a representative ganglioside, $\quad \mathrm{GM}_{1} \quad$ (galactosyl- $N$-acetylgalactosaminyl ( $N$-acetylneuraminyl)galactosylglucosyl ceramide) (Fig. 2), which — being important membrane components of lipid rafts (sphingomyelin) and tumor membranes (gangliosides) - may play a crucial role in attracting the drugs selectively to antineoplastic cells.
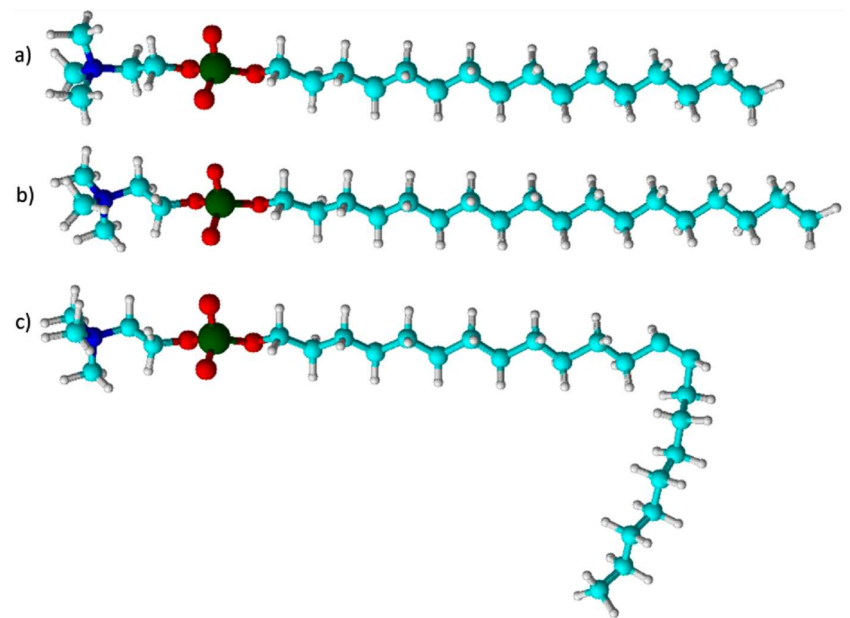

Fig. 1. Chemical structure of the investigated APCs: $\mathrm{HePC}(\mathrm{a}), \mathrm{OcPC}(\mathrm{b})$ and $\operatorname{ErPC}(\mathrm{c})$.

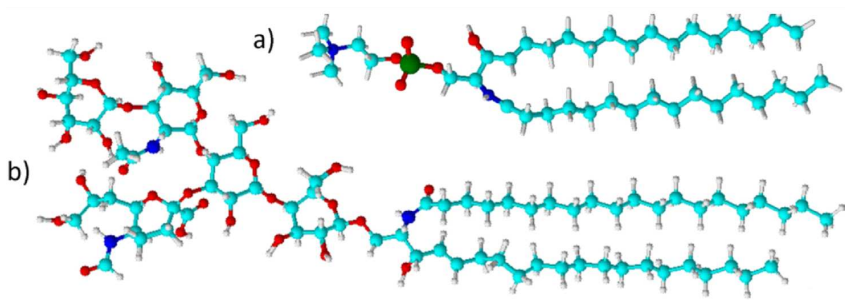

Fig. 2. Chemical structure of SM (a) and $\mathrm{GM}_{1}$ (b).
To reach our goal we have applied the Langmuir monolayer technique [18], which serves as a useful two-dimensional model of biological membranes [19, 20], to examine interactions between biomembrane components and the drugs. The interactions have been analyzed qualitatively with the compression modulus $\left(C_{\mathrm{S}}^{-1}\right)$ and mean molecular area $\left(A_{12}\right)$ as well as quantitatively by calculating the excess free enthalpy $\left(\Delta G^{\mathrm{exc}}\right)$ of mixing. The details concerning the calculations of the above-mentioned parameters of interactions can be found elsewhere [21].

\section{Experimental}

The investigated antitumor alkylphosphocholines: hexadecylphosphocholine (HePC), octadecylphosphocholine (OcPC) and erucylphosphocholine (ErPC) were obtained from Avanti Polar Lipids, A.G. Scientific and Aeterna Zentaris GmbH, respectively. Both investigated sphingolipids (sphingomyelin and ganglioside $\mathrm{GM}_{1}$ ) were purchased from Avanti Polar Lipids. All the studied substances were kept in closed bottles in the freezer and used without further purification. The spreading solutions were prepared by dissolving appropriate weights of substances in spectroscopic grade chloroform/methanol $(9: 1 \mathrm{v} / \mathrm{v})$ mixture. Langmuir monolayers were obtained by spreading an aliquot of the above-mentioned solutions (concentration of $c a .0 .5 \pm 0.2 \mathrm{mg} / \mathrm{mL}$ ) with a Hamilton microsyringe (precise to $\pm 2 \mu \mathrm{L}$ ) onto the surface of ultrapure water. Mixed solutions were obtained by mixing proper volumes of respective stock solutions. Firstly, binary mixtures composed of SM and selected APCs were prepared $(\mathrm{SM} / \mathrm{HePC}, \mathrm{SM} / \mathrm{OcPC}$ and $\mathrm{SM} / \mathrm{ErPC}$; $\mathrm{APCs}$ ratio ranged from 0 to 1 with the increment of 0.1 ) and then - in analogy - mixtures with $\mathrm{GM}_{1}$ were made. The film compression (with the barrier speed of $50 \mathrm{~cm}^{2} / \mathrm{min}$ ) was initiated 5 min after spreading to ensure total evaporation of the solvent. The surface pressure-area $(\pi-A)$ isotherms were obtained with a 611 Langmuir-Blodgett trough (Coventry, U.K.) (total area $=600 \mathrm{~cm}^{2}$ ) placed on an anti-vibration table. Surface pressure was measured to within $0.1 \mathrm{mN} / \mathrm{m}$ using a Wilhelmy plate made from ashless chromatography paper. The temperature of the aqueous subphase was held constant to $20^{\circ} \mathrm{C} \pm 0.1^{\circ} \mathrm{C}$ by a circulating water system from Julabo.

\section{Results and discussion}

Three antitumor phosphocholines (APCs), namely hexadecylphosphocholine (HePC), octadecylphosphocholine (OcPC) and erucylphosphocholine (ErPC) have already been studied alone as well as in mixtures with two main membrane lipids, i.e. cholesterol and phosphatidylcholines (DPPC and POPC) [12]. In this paper we have extended our study to mixtures with sphingolipids: sphingomyelin (SM) and ganglioside $\mathrm{GM}_{1}$. The isotherms for both sphingolipids [22-24] as well as APCs [12] are known and our results are in a good agreement 
with the data already published. Namely, both investigated sphingolipids behave similarly in monolayers at the air/water interface (Figs. 3-4 a-c), i.e. they form condensed films, as proved by the compression moduli $\left(C_{\mathrm{S}}^{-1}=-A(\mathrm{~d} \pi / \mathrm{d} A)[25]\right)$ values (reaching maximum values of $285 \mathrm{mN} / \mathrm{m}$ for $\mathrm{SM}$ monolayers and $180 \mathrm{mN} / \mathrm{m}$ for $\left.\mathrm{GM}_{1}\right)$ and collapsing at the surface pressure $\left(\pi_{\text {coll }}\right)$ of about $69 \mathrm{mN} / \mathrm{m}(\mathrm{SM})$ and $65 \mathrm{mN} / \mathrm{m}\left(\mathrm{GM}_{1}\right)$.

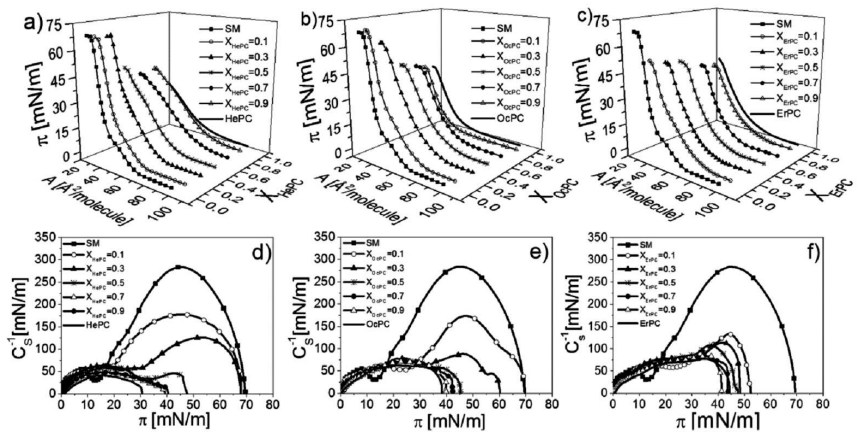

Fig. 3. Surface pressure $(\pi)$-area $(A)$ isotherms for APCs, sphingomyelin and their mixtures $(\mathrm{a}-\mathrm{c})$. Compression modulus $\left(C_{\mathrm{s}}^{-1}\right)$-surface pressure $(\pi)$ dependences $(\mathrm{d}-\mathbf{f})$

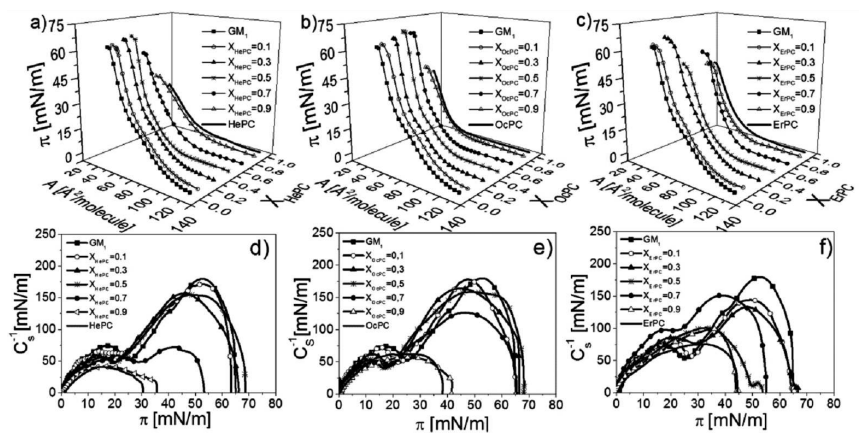

Fig. 4. Surface pressure $(\pi)$-area (A) isotherms for APCs, ganglioside $\mathrm{GM}_{1}$ and their mixtures $(\mathrm{a}-\mathrm{c})$. Compression modulus $\left(C_{\mathrm{s}}^{-1}\right)$-surface pressure $(\pi)$ dependences $(d-f)$.

However, detailed analysis of the shape and localization of isotherms for both sphingolipids points out some differences. Namely, larger molecular areas for the lift-off for pure $\mathrm{GM}_{1}$ monolayer (ca. $135 \AA^{2} /$ molecule) as compared to SM film (ca. $78 \AA^{2} /$ molecule) indicates a lower ordering and more expanded character of ganglioside monolayers, resulting from the structure of the ganglioside headgroup, which is much bulkier than the polar moiety of sphingomyelin and, additionally, it is negatively charged. In consequence, the electrostatic repulsions hinder a close packing of $\mathrm{GM}_{1}$ molecules and ordering of the hydrophobic chains in the monolayer. In the course of the isotherms recorded for both sphingolipids at $20^{\circ} \mathrm{C}$, a phase transition between $\mathrm{LE}$ and LC state is observed (at $c a .13 \mathrm{mN} / \mathrm{m}$ for $\mathrm{SM}$ and $c a .20 \mathrm{mN} / \mathrm{m}$ for $\left.\mathrm{GM}_{1}\right)$. This transition is visible as a clear minimum on $C_{\mathrm{s}}^{-1}=f(\pi)$ plots (Figs. 3-4, d-f).

As far as the isotherms of pure APCs are concerned, they form liquid-type films, without any visible transition in the course of their isotherms. As it has already been reported in our previous paper [12], their monolayers become more condensed (higher maximum $C_{\mathrm{s}}^{-1}$ values) and more stable (higher values of $\pi_{\text {coll }}$ ) upon increase of the hydrocarbon chain length.

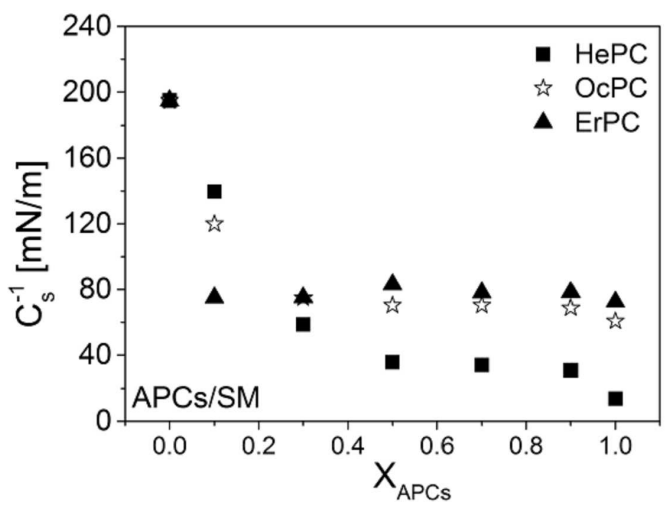

Fig. 5. Maximum values of the compression modulus $\left(C_{\mathrm{s}}^{-1}\right)$ due to incorporation of APCs into the monolayers of $\mathrm{SM}$ at $30 \mathrm{mN} / \mathrm{m}$.

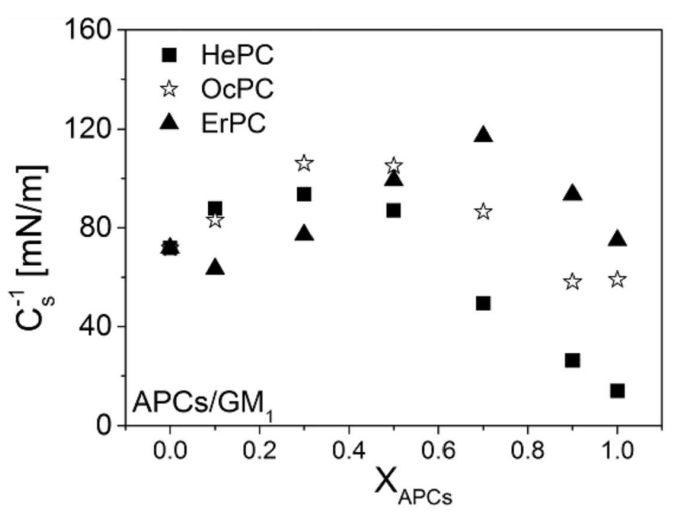

Fig. 6. Maximum values of the compression modulus $\left(C_{\mathrm{s}}^{-1}\right)$ due to incorporation of APCs into the monolayers of $\mathrm{GM}_{1}$ at $30 \mathrm{mN} / \mathrm{m}$.

The isotherms recorded for $\mathrm{HePC}, \mathrm{OcPC}$ and $\mathrm{ErPC}$ mixed with membrane sphingolipids lipids are presented in Figs. 3-4, a-c. The addition of APCs into the SM and $\mathrm{GM}_{1}$ monolayers influences the organization of films from both sphingolipids, however, in a different way (Figs. 5, 6). In the case of sphingomyelin monolayer (which is more condensed as compared to the film formed by $\mathrm{GM}_{1}$ ), the incorporation of APC molecules in small amounts significantly decreases the compression modulus of the film. However, upon further addition of APCs (above $X_{\mathrm{APC}}=0.3$ ), the film fluidity remains nearly constant, indicating that SM monolayer is no longer accessible for APC molecules. Different phenomenon is ob- 
served for $\mathrm{GM}_{1}$ monolayer, which is more fluid, i.e. APC molecules gradually incorporate into the film, which becomes more packed and finally, for high proportion of the drug, the organization of the film is disturbed, which is reflected in a significant decrease of the compression modulus. Such an interesting effect of ganglioside, condensing a monolayer in small amounts and fluidizing in higher concentration was also reported for DPPC-GM mixture [26].

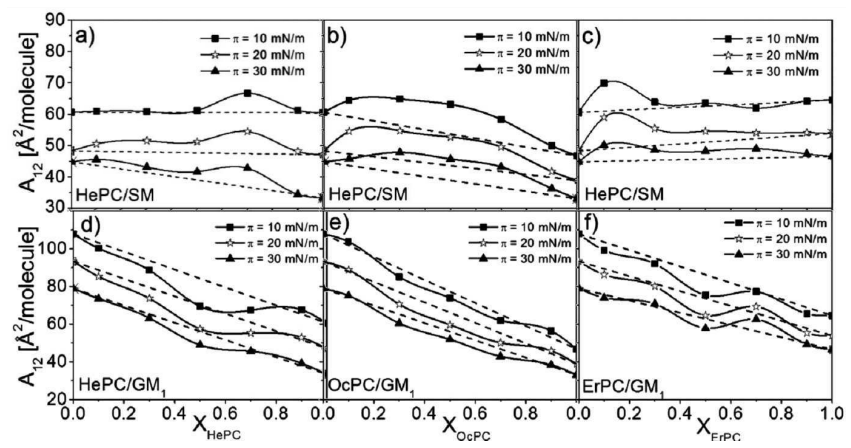

Fig. 7. Mean molecular area $\left(A_{12}\right)$ vs. mixed film composition $\left(X_{\mathrm{APCs}}\right)$ plots for mixtures of APCs with investigated sphingolipids at different constant surface pressures.

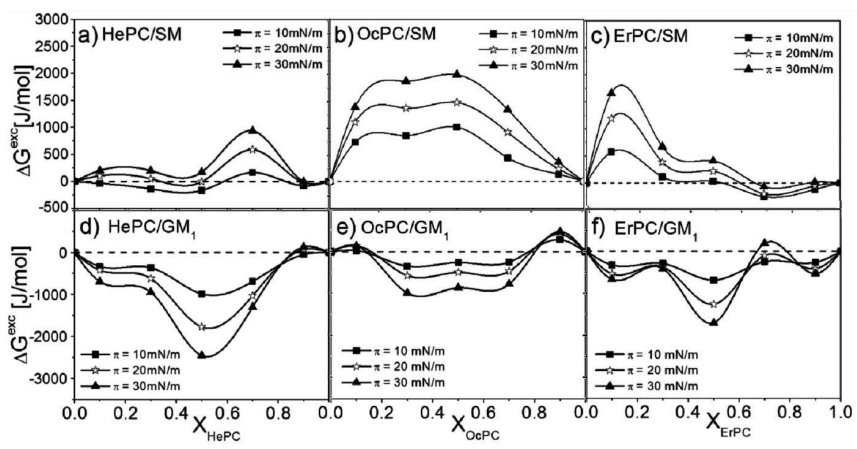

Fig. 8. Excess free energy of mixing $\left(\Delta G^{\text {exc }}\right)$ vs. mixed film composition $\left(X_{\mathrm{APCs}}\right)$ plots for mixtures of APCs with investigated sphingolipids at different constant surface pressures.

In order to compare the effect of APCs addition on the area per molecule in both mixed systems, the mean molecular areas $\left(A_{12}\right)$ were calculated and presented in Fig. 7. The observed deviations from ideality are different for systems containing SM versus $\mathrm{GM}_{1}$. The mean area values for $\mathrm{APCs} / \mathrm{SM}$ mixtures are - in general positive (although the maximum value from linearity occurs for different stoichiometry, depending on particular $\mathrm{APC}$ ), while for $\mathrm{APCs} / \mathrm{GM}_{1}$ monolayers the contraction of area per molecule (reflected in negative values of $A_{12}$ ) was found. Positive deviations indicate that the interactions between APCs and SM molecules in mixed monolayers are less attractive (or more repulsive) as compared to those existing in one component films (APC-APC or SM-SM). On the contrary, the interactions between
APCs and $\mathrm{GM}_{1}$ are more attractive in their mixed films versus those between like molecules in their pure monolayers.

The affinity of APCs to the investigated membrane sphingolipids can be quantified with the excess free energy of mixing values $\left(\Delta G^{\text {exc }}\right)$ (Fig. 8). The incorporation of APCs into sphingomyelin monolayer is unfavourable due to the decrease of attractive interactions between components, which may lead to phase separation in mixed monolayers, while the attractive, favourable interactions between APCs and $\mathrm{GM}_{1}$ reflect in negative values of $\Delta G^{\text {exc }}$. The presence of a minimum at 1:1 proportion for mixtures with HePC and ErPC implies that the strongest molecular interactions, leading to the highest films stability, occurred at that very composition. For mixed films composed of $\mathrm{OcPC}$ and $\mathrm{GM}_{1}$, there is no minimum in $\Delta G^{\text {exc }}$ values and the strength of attractive interactions is comparable in a broad composition range $\left(x_{\mathrm{OcPC}}=0.3-0.7\right)$

Different affinity of interacting molecules can also be analysed with geometric packing of molecules, which is expressed in term of a dimensionless critical packing parameter $s$ (defined as: $s=\frac{V}{a l_{c}}[27,28]$ ) that depends on the head group area $a$, volume $V$, and critical length $l_{\mathrm{c}}$ of the hydrocarbon chain. Our calculations [21] revealed that for APCs, $s<1$, which indicates their conical shapes (HePC and OcPC - cone; ErPC - truncated cone). The thermodynamic analysis for mixtures of APCs with cholesterol and phosphatidylcholines (PCs), performed earlier [12], proved the existence of strong attractive interactions with cholesterol, while weak repulsive interactions were observed for mixtures with PCs. These results agree well with the analysis of the geometry of interacting molecules. Namely, conically shaped APCs ensure favorable packing with cholesterol, which is of opposite geometry (inverted cone, $s>1$ ), while the arrangement with conical PCs cannot be expected to be so favorable.

In this work we observe repulsive interactions between APCs and SM, which can be understood basing on the fact that SM has a cylinder shape [29]; therefore in this case shapes complementarity is not optimal. However, mixtures with $\mathrm{GM}_{1}$ were found to be attractive, although the shape of both APCs and $\mathrm{GM}_{1}$ is similar (conical) [30]. It should be stressed, however, that molecular shapes are estimated from the values of critical packing parameter, which does not take into account specific interactions between mixture components, i.e. it does not consider electrostatic effects, alterations in hydration, chain motion, etc. In APCs-GM1 monolayers favorable packing can be mainly due to electrostatic forces between positively charged choline group of the APC molecule and negatively charged sialic acid residue in GM1, which prevails over the shape incomplementarity between both molecules.

\section{Conclusions}

In this contribution, the Langmuir monolayer technique has been applied to investigate the interactions be- 
tween three representatives of new generation anticancer drugs APCs: HePC, OcPC, and ErPC and two membrane sphingolipids: $\mathrm{SM}$ and $\mathrm{GM}_{1}$, aiming at finding a molecular target for APCs, which can selectively attract them to tumor cells. The thermodynamic analysis of the obtained isotherms proved that the interactions between SM and APCs are weak and repulsive, while strong affinity of the drugs was found to the ganglioside $\mathrm{GM}_{1}$. This implies that gangliosides, which overexpress in cancerous membrane, may be considered as molecular targets, attracting APCs molecules to antineoplastic cells and therefore can be responsible for a high selectivity observed for these drugs.

The affinity of the investigated APCs to membrane sphingolipids was also analyzed basing on geometry of interacting molecules. Similar molecular shapes of both studied sphingolipids and APCs suggest unfavorable packing of molecules in monolayers, which should result in weak and repulsive interactions between molecules. This prediction was proved to be correct for APCssphingomyelin system, however, it did not work out for mixtures with $\mathrm{GM}_{1}$, which were found to be attractive. This discrepancy was ascribed to electrostatic forces between APCs and $\mathrm{GM}_{1}$, which prevails over the shape incomplementarity between both molecules. No significant differences in the strength of interactions with sphingolipids were found upon increase of the hydrocarbon chain length of APC molecule (HePC versus $\mathrm{OcPC}$ ) or by introducing of the unsaturated bond (ErPC).

\section{Acknowledgments}

Aeterna Zentaris GmbH (Frankfurt, Germany) is gratefully acknowledged for providing a sample of erucylphosphocholine for investigations. The research was carried out with the equipment (Langmuir trough) purchased thanks to the financial support of the European Regional Development Fund in the framework of the Polish Innovation Economy Operational Program (contract no. POIG.02.01.00-12-023/08). The financial support from the National Science Centre (NCN, Poland, grant No. UMO-2012/07/N/NZ1/00005) is gratefully acknowledged.

\section{References}

[1] C. Gajate, F. Mollinedo, Curr. Drug Metab. 3, 491 (2002).

[2] T. Wieder, W. Reutter, C.E. Orfanos, C.C. Geilen, Prog. Lipid Res. 38, 249 (1999).

[3] C. Unger, E.A.M. Fleer, J. Kötting, W. Neumüller, H. Eibl, Prog. Exp. Tumor Res. 34, 25 (1992).

[4] H. Eibl, C. Unger, Cancer Treat. Rev. 17, 233 (1990).

[5] V. Jendrossek, I. Müller, H. Eibl, C. Belka, Oncogene 22, 2621 (2003).
[6] C. Gajate, R.I. Fonteriz, C. Cabaner, G. AlvarezNoves, Y. Alvarez-Rodriguez, M. Modolell, F. Mollinedo, Int. J. Cancer 85, 674 (2000).

[7] S.M. Johnson, R. Robinson, Biochim. Biophys. Acta 558, 282 (1979).

[8] G. Agatha, R. Hafer, F. Zintl, Cancer Lett. 173, 139 (2001).

[9] M. Sok, M. Šentjurc, M. Schara, Cancer Lett. 139 215 (1999).

[10] M.R. Freeman, K.R. Solomon, J. Cell. Biochem. 91, 54 (2004).

[11] S. Riedl, D. Zweytick, K. Lohner, Chem. Phys. Lipids 164, 766 (2011).

[12] A. Wnętrzak, K. Łątka, P. Dynarowicz-Łątka, J. Membrane Biology 246, 453 (2013).

[13] M. Rybczyńska, M. Spitaler, N.G. Knebel, G. Boeck, H. Grunicke, J. Hofmann, Biochem. Pharm. 62, 765 (2001).

[14] A.H. Van der Luit, S.R. Vink, J.B. Klarenbeek, D. Perrissound, E. Solary, M. Verheij, W.J. Blitterswijk, Mol. Cancer Ther. 6, 2337 (2007).

[15] T. Nieto-Miguel, C. Gajate, F. Mollinedo, J. Biol. Chem. 281, 14833 (2006).

[16] A. Ausili, A. Torrecillas, F.J. Aranda, F. Mollinedo, C. Gajate, S. Corbalán-Garcia, A. de Godos, J.C. Gómez-Fernández, J. Phys. Chem. B 112, 11643 (2008).

[17] R.G. Fish, Med. Hypotheses 46, 140 (1996).

[18] G.L. Gaines Jr, Insoluble Monolayers at Liquid-Gas Interfaces, Interscience, New York 1966.

[19] R. Maget-Dana, Biochim. Biophys. Acta 1462, 109 (1999).

[20] C. Peetla, A. Stine, L. Labhasetwar, Mol. Pharmaceut. 6, 1264 (2009).

[21] P. Dynarowicz-Łątka, K. Kita, Adv. Coll. Interface Sci. 79, 1 (1999).

[22] J.M. Smaby, H.L. Brockman, R.E. Brown, Biochemistry 33, 9135 (1994).

[23] R. Wüstneck, N. Wüstneck, D.O. Grigoriev, U. Pison, R. Miller, Colloids Surf. B 15, 275 (1999).

[24] M. Roefzaad, T. Kluner, I. Brand, Phys. Chem. Chem. Phys. 11, 10140 (2009).

[25] J.T. Davies, E.K. Rideal, Interfacial Phenomena, Academic Press, New York 1963.

[26] S.L. Frey, E.Y. Chi, C. Arratia, J. Majewski, K. Kjaer, K.Y.C. Lee, Biophys. J. 94, 3047 (2008).

[27] J.N. Israelachvili, Intermolecular and Surface Forces, Academic Press, London 1985.

[28] J.N. Israelachvili, S. Marcelja, R.G. Horn, Q. Rev. Biophys. 13, 121 (1980).

[29] P.A. Janmey, P.K.J. Kinnunen, Trends Cell Biol. 16 , 538 (2006).

[30] M.A. Perillo, N.J. Scarsdale, R.K. Yu, B. Maggio, Proc. Natl. Acad. Sci. USA 91, 10019 (1994). 